\title{
Wildlife Observation Robot Using RF
}

\author{
Mr. Atul Thorat ${ }^{1}$, Miss. Hemlata Powar ${ }^{2}$, Mr.Sagar Ingale ${ }^{3}$, Miss. Shital Surve ${ }^{4}$ \\ Student, Dept of E\&TC, DACOE Karad, Kolhapur University, India \\ Assistant Professor, Dept of E\&TC, DACOE Karad, Kolhapur University, India
}

\begin{abstract}
Here the wildlife observation robot with night vision capability system makes use of RF remote joystick to operate the robot on which the night vision camera is being mounted $360 \mathrm{deg}$. This allows the user to control the robotic vehicle wirelessly and get required angled of these wild animals. This video is recorded and can be viewed on PC for reference later. So wildlife observers can now safely get close footage of wild animals by operating this robotic vehicle without any threat from a safe distance. This system consists of an 8051 family microcontroller CPU unit used for processing user sent through the RF transmitter circuit. These signals are received by the RF receiver mounted on the robot. The microcontroller then processes this data and passes on signals to driver motors to control the robot. The driver motors now in turn operate the motors by providing required signal outputs to drive the vehicle movement motors. Also when the microcontroller receives the camera directional change signal, it then forwards this signal to the camera motor in order to achieve required camera angle. Thus this wildlife observation robot with night vision capability system helps to get a closer view of wildlife with the help of RF remote joystick.
\end{abstract}

Keywords: RF module, motor driver L293D, Night vision camera, dc motor, night vision.

\section{INTRODUCTION}

Project makes use of this innovative system in order to get tags are used to track their motion using radio telemetry close footage of wild animals using 360 deg cameras. As [1].

with the help of this system the user doesn't have to go Mine Rover- It was developed in 2005 abandoned minesclose to the wild animals in order to get the close footage remnants of old west mining booms -closely guard their of movement of animals. Here the wildlife observation secrets in the forgotten corners of Arizona's backcountry. robot with night vision capability system makes use of RF What's concealed just around that bend in the tunnel are remote joystick to operate the robot on which the night the inevitable questions those hikers and others ask when vision camera is being mounted $360 \mathrm{deg}$. This allows the they stumble across these slumbering relics. Those can be user to control the robotic vehicle wirelessly and get dangerous questions[2]. Crumbling walls and ceilings that required angled of these wild animals. This video is threaten to collapse at the slightest touch; hidden vertical recorded and can be viewed on PC for reference later. So shafts; poisonous gases; wildlife lurking inside are just wildlife observers can now safely get close footage of wild some of the dangers that prevent the non suicidal from animals by operating this robotic vehicle without any exploring. Still the question remains: what's inside threat from a safe distance. This system consists of an They've built an 18 inch long, radio controlled rover to do 8051 family microcontroller CPU unit used for processing the looking for them it's equipped with a power full user sent through the RF transmitter circuit. These signals search light to explore the mines dark recesses and a pan are received by the RF receiver mounted on the robot. and tilt video camera to send images back to their laptop The microcontroller then processes this data and passes on computer. Jessica Dooley and Keith Brock made the signals to driver motors to control the robot. The driver ground rover to tour a mine [3].

motors now in turn operate the motors by providing required signal outputs to drive the vehicle movement motors. Also when the microcontroller receives the camera directional change signal, it then forwards this signal to the camera motor in order to achieve required camera angle. Thus this wildlife observation robot with night vision capability system helps to get a closer view of wildlife with the help of RF remote joystick.

\section{II.LITERATURE REVIEW}

The driver motors now in turn operate the motors by providing desired signal outputs to drive the vehicle movement motors. Also when the microcontroller receives the camera directional change signal, it then forwards this signal to the camera motor in order to achieve desired camera angle [4]

\section{SYSTEM DESCRIPTION}

The basic block diagram of wildlife observation system Usage of sensor networks in monitoring, tracking and detection of different wildlife species is a common practice that is carried on. For small animals and birds, RF using RF system is shown in the above figure. Mainly this block diagram consists of the following essential blocks. 8051 series Microcontroller 
IARJSET

RF Module

Antenna

Motor driver IC

Wireless Camera

\section{- Transmitter}

It consists of an RF transmitter module with encoder and joystick. It requires $9 \mathrm{v}$ power supply to operate. With the help transmitter section we can control our robot. to move left, right, forward ,backward this section is used. Transmitter also transfers command from four buttons to receiver. Transmitter can control the camera direction which is placed on the robot.

\section{- Receiver}

This section has consists of main spy robot. The night vision camera placed on this section at 180 degree. In this there are three servo motors are require two for moving robot and one for the moving camera. Microcontroller 8051 interface with RF receiver requires $12 \mathrm{v}$ battery supplies to operate. RF receiver receives $\mathrm{RF}$ commands from the transmitter and decodes it using decoder \& performs as requires. To drive motors we require motor driver ic. The movement of animals captured by camera is then displayed on the PC for reference.



Fig-transmitter

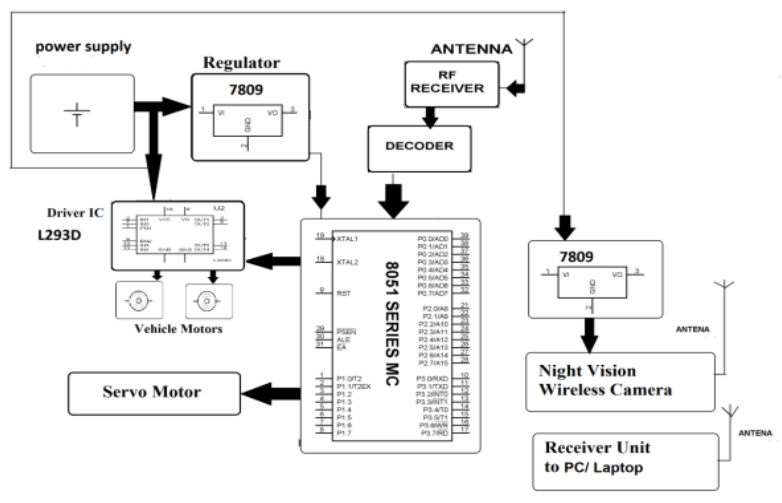

- RF module

Fig-receiver

This radio frequency (RF) transmission system employs Amplitude Shift Keying (ASK) with transmitter/receiver (TX/Rx) pair operating at $434 \mathrm{MHz}$ The transmitter encoder module takes serial input and transmits these signals through RF.
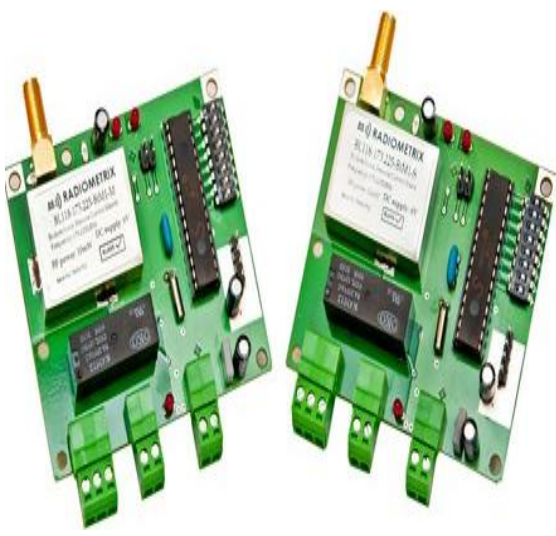

The transmitted signals are received by the receiver decoder module placed away from the source of transmission. The system allows RF communication one way communication between two nodes, namely, transmission and reception. The RF module has been used in conjunction with a set of four channel encoder and decoder ICs. Here HT12E \& HT12D have been used as encoder and decoder. The encoder converts the parallel inputs (from the remote joystick) into serial set of signals. These signals are serially transferred through RF to the reception or receiver. The decoder is used after the RF receiver to decode the serial format and retain the original signals as outputs [5].

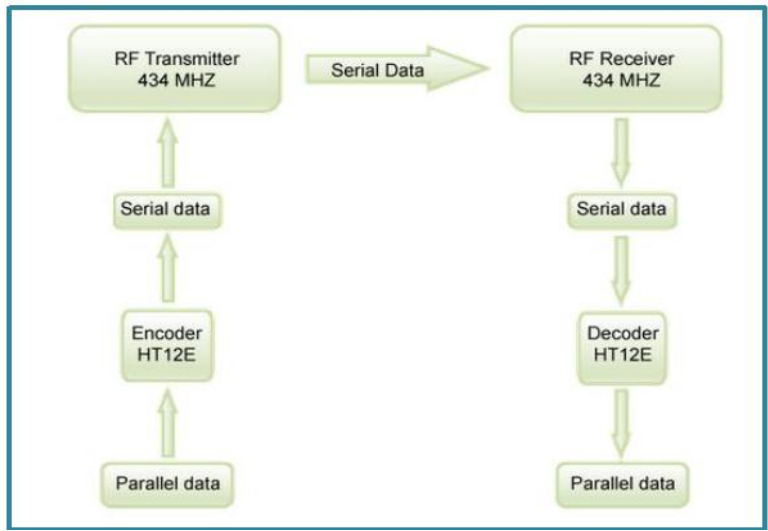

Motor driver IC L293D

\section{- Features}

Wide Supply-Voltage Range: 4.5 V to $36 \mathrm{~V}$

Separate Input-Logic Supply

Internal ESD Protection

High-Noise-Immunity Inputs

Output Current 1 A Per Channel (600 mA for

L293D)

\section{- Applications \\ Stepper Motor Drivers \\ DC Motor Drivers}

Latching Relay Drivers

\section{MOTOR}

A DC motor is a mechanically commutated electric motor powered from direct current (DC). The stator is stationary 
in space by definition and therefore its current. The current $8 \mathrm{~K}$ Bytes of Programmable Flash Memory. in the rotor is switched by the commutate or to also be $4.0 \mathrm{~V}$ to $5.5 \mathrm{~V}$ Operating Range. stationary in space. DC motors have a rotating armature $256 \times 8$-bit Internal RAM. winding but non -rotating armature magnetic field and a 32 Programmable I/O Lines. static field winding or permanent magnet. Different Three 16-bit Timer/Counters. connections of the field and armature winding provide Eight Interrupt Sources. different inherent speed/torque regulation characteristics. Full Duplex UART Serial Channel. The speed of a DC motor can be controlled by changing Low-power Idle and Power-down Modes. the voltage applied to the armature or by changing the field current. Modern DC motors are often controlled by power electronics systems called DC drives[6].

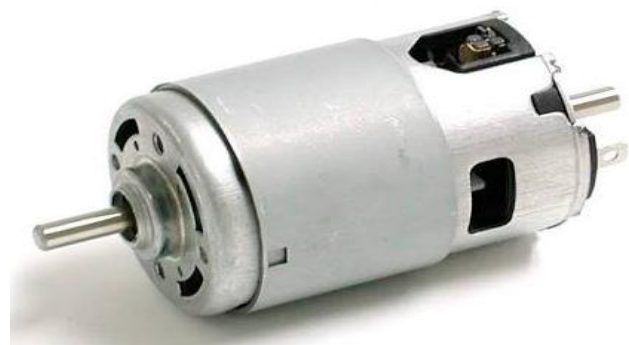

Fig-DC motor

\section{MICROCONTROLLER 8051}

Microcontroller (MC) may be called computer on chip since it has basic features of microprocessor with internal ROM, RAM, Parallel and serial ports within single chip. Or we can say microprocessor with memory and ports is called as microcontroller. This is widely used in washing machines ved player, microwave oven, and robotics or in industries. Microcontroller can be classified on the basis of their bits processed like8bit MC, 16bit MC. 8 bit microcontroller means it can read write and process 8 bit data. Ex. 8051 microcontroller. Basically 8 bit specifies the size of data bus. 8 bit microcontroller means 8 bit data can travel on the data bus or we can read, write process 8 bit data.

\section{Pin diagram of $\mathbf{8 0 5 1}$}

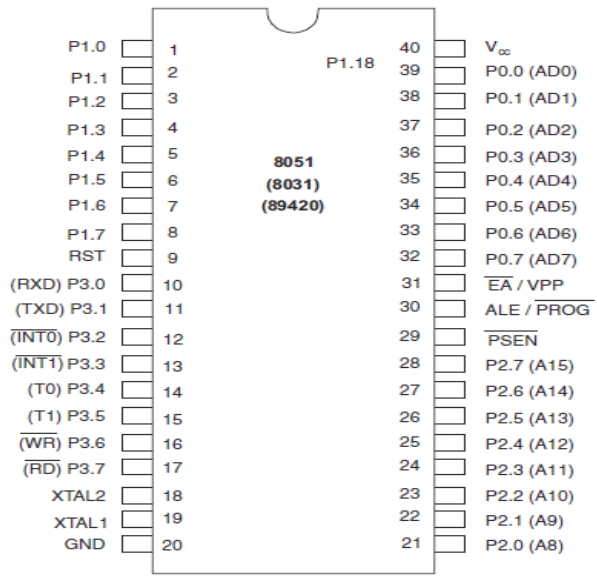

\section{CONCLUSION}

In this project we have conclude that the close footage and secure data can be obtain without any threat. Wildlife observation robot is more reliable system than older system to obtain movement of animals.

\section{ACKNOWLEDGMENT}

We would like to thank sincerely to our guide Miss. H.R. Powar and thanks for endless support and motivation for her invaluable guidance, constant assistance, support, endurance and constructive suggestions for the betterment of this project work.

\section{REFERENCES}

[1] International Journal of Innovative Research in Science, Engineering and Technology Department of TCE, Don Bosco Institute of Technology, Bangalore, Karnataka, India3456

[2] International Journal of Innovative Research in Science, Engineering and Technology Snehal D. Patil, Archana A. Hatkar Student, Dept. of E\&TC, SVIT Nasik, Pune University, Maharashtra, India Associate Professor, Dept. of E\&TC, SVIT Nasik, Pune University, Maharashtra, India

[3] Nevon developer's site Nevon projects.com

[4] Xiaohan Liu, Tao Yang, Baoping Yan "Research on the Architecture of Wildlife Observation and Communication System" Computer Network Information Center (CNIC) Chinese Academy of Sciences Beijing, China IEEE 2015 [2] [5] William P. Bennett, Jr., Mehmet C. Vuran , Matthew B. Dwyer, Sebastian Elbaum , Anne Lacy, Mike Engels, Walter Wehtje "Sensing Through the Continent: Towards Monitoring Migratory Birds Using Cellular Sensor Networks" Department of Computer Science and Engineering University of Nebraska - Lincoln, Lincoln, NE.IEEE April 2012.

[6] Gilberto Antonio Marcon dos Santos1Alex Ke, Han Lin, Curt Schurgers, Albert Lin and Ryan Kastner, Zachary Barnes "Small Unmanned Aerial Vehicle System for Wildlife Radio Collar Tracking" University of California, San Diego , Eric Lo, Bryan Ritoper, Lauren Nishizaki, Xavier Tejeda2. 2014 IEEE 11th International Conference on Mobile Ad Hoc and Sensor Systems. Pg no 761-766

\section{- Features of 8051}

Fully TTL Compatible.

4 8bit bidirectional I/O ports. 